\title{
Mediating Role of Anger Rumination in the Associations between Mindfulness, Anger-In, and Trait Anger
}

\author{
Masaya Takebe1 ${ }^{1}$, Fumito Takahashi' ${ }^{2}$, Hiroshi Sato ${ }^{3}$ \\ ${ }^{1}$ Graduate School of Psychology, Kansai University, Osaka, Japan \\ ${ }^{2}$ Institute of Education, Shinshu University, Nagano, Japan \\ ${ }^{3}$ Faculty of Sociology, Kansai University, Osaka, Japan \\ Email: g7yatto.zrm99@gmail.com
}

Received 7 May 2015; accepted 19 June 2015; published 23 June 2015

Copyright (C 2015 by authors and Scientific Research Publishing Inc.

This work is licensed under the Creative Commons Attribution International License (CC BY). http://creativecommons.org/licenses/by/4.0/

(c) (7) Open Access

\begin{abstract}
While there are several studies that have investigated the specific relationships between mindfulness, anger rumination, anger-in, and trait anger, no study has investigated all these relationships simultaneously. Therefore, this study examined all relationships between these variables employing a cross-sectional design. Consistent with previous research, we hypothesized that (1) mindfulness will reduce anger rumination, (2) anger rumination will facilitate anger-in and trait anger, and (3) mindfulness will indirectly alleviate anger-in and trait anger through reducing anger rumination. The total of 366 Japanese undergraduates $(M=20.18 ; S D=2.27 ; 51.1 \%$ female; 48.9\% male) had completed the questionnaires. The results of this study revealed that (1) mindfulness reduced anger rumination, (2) anger rumination facilitated anger-in and trait anger, and (3) mindfulness indirectly alleviated anger-in and trait anger through reducing anger rumination. Although the mediating role of anger rumination was discussed in previous study, it was not based on data. The current study verifies the assumptions by previous study and suggests that anger rumination may be a key factor in treating problems of anger-in and trait anger. We provide new evidence contributing to improved treatment options by specifying the relationships between mindfulness, anger rumination, anger-in, and trait anger.
\end{abstract}

\section{Keywords}

Anger Rumination, Anger-In, Trait Anger, Mindfulness, Mediator 


\section{Introduction}

Mindfulness is defined as the quality of consciousness or awareness that arises through intentionally attending to the present moment experience in a nonjudgmental and accepting way (Kabat-Zinn, 1994). Mindfulness is effective for anger problems. Several studies have suggested that mindfulness exerts indirect effects in alleviating anger (e.g., Lane, Seskevich, \& Pieper, 2007). Silva (2007) examined the efficacy of mindfulness-based cognitive therapy (MBCT) for anger problem and indicated that MBCT had a positive effect on people's life and helped them deal with anger. In recent years, mindfulness has become the focus of attention as a treatment of anger.

In addition to the efficacy of mindfulness, factors mediating its effects on anger problems have been thoroughly investigated, especially the relationships with rumination (Wright, Day, \& Howells, 2009). Borders, Earleywine, \& Jajodia (2010) investigated the relationships between mindfulness, anger problems (e.g., aggressiveness), and rumination. Based on their results, Borders et al. (2010) suggested that mindfulness alleviated hostility and aggressiveness by reducing rumination. In fact, mindfulness indirectly affects anger problems through rumination.

There is good evidence that rumination is associated with both anger-in (Martin \& Dahlen, 2005) and trait anger (Owen, 2011). Anger-in is a tendency to suppress anger and is related to various other problems, such as social anxiety (Trew \& Alden, 2009) and cardiovascular disease (Jorgensen \& Kolodziej, 2007). Trait anger is defined as "in terms of individual differences in the disposition to perceive a wide range of situations as annoying or frustrating and by the tendency to respond to such situations with elevations in state anger” (Spielberger, 1999: p.1).

Anger rumination is a more specific style of rumination, which is defined as a tendency to engage in unintentional re-occurring thoughts about anger episodes (Sukhodolsky, Golub, \& Cromwell, 2001). Sukhodolsky et al. (2001) constructed the Anger Rumination Scale (ARS) to measure the cognitive processes of individuals experiencing anger. Although several self-report measures to evaluate cognitive responses to emotional experiences have been developed in the context of self-regulation of depressed mood (e.g., Nolen-Hoeksema \& Morrow, 1991), the ARS permits measuring cognitive response and anger process directly.

While there are several studies that have investigated the specific relationships between mindfulness, anger rumination, anger-in, and trait anger, no study has investigated all these relationships simultaneously. Therefore, this study examined all relationships between these variables employing a cross-sectional design. Consistent with previous research, we hypothesized that (1) mindfulness will reduce anger rumination, (2) anger rumination will facilitate anger-in and trait anger, and (3) mindfulness will indirectly alleviate anger-in and trait anger through reducing anger rumination. We provide new evidence contributing to improved treatment options by specifying the relationships between mindfulness, anger rumination, anger-in, and trait anger.

\section{Methods}

\subsection{Sample and Procedures}

This study was approved by the ethics board of human research of Shinshu University. Questionnaires were completed by 446 university students resulting in 366 (82.1\%) samples with valid responses. All individuals provided informed consent. Of the participants, 51.1\% identified themselves as men and $48.9 \%$ as women. The mean age of the participants was 20.18 years $(S D=2.27)$.

\subsection{Measurements}

\subsubsection{Mindfulness}

The Japanese Version of the Five Facet Mindfulness Questionnaire (FFMQ; Sugiura, Sato, Ito, \& Murakami, 2012) was used to assess mindfulness. The FFMQ (Baer, Smith, Hopkins, Krietemeyer, \& Toney, 2006) is a 39-item measure of various aspects of mindfulness including non-reactivity to inner experience, observing/ attending to sensations, acting with awareness, describing, and non-judging of experience. Participants respond on a 5-point Likert-type scale that ranges from 1 (never or very rarely true) to 5 (very often or always true). The FFMQ has adequate convergent and discriminant validity and reliability (Baer et al., 2006). The alpha coefficient in this sample was 0.78 .

\subsubsection{Anger Rumination}

The Japanese Version of the Anger Rumination Scale (ARS; Hatta, Ohbuchi, \& Hatta, 2013) was used. The 
Anger Rumination Scale is a 19-item self-report questionnaire examining the degree to which individuals tend to focus on angry moods (e.g., "Whenever I experience anger, I keep thinking about it for a while.”). Items utilize a Likert scale ranging from 1 (almost never) to 4 (almost always). The ARS has adequate convergent and discriminant validity and reliability (Sukhodolsky et al., 2001). The alpha coefficient in this sample was 0.94 .

\subsubsection{Anger}

The Japanese Version of the State Trait Anger Expression Inventory (STAXI; Suzuki \& Haruki, 1994) was used. The STAXI (Spielberger, 1988) is a 44-item measure that assesses the experience and expression of anger. Items are rated on a 4-point Likert-type scale from 1 (almost never) to 4 (almost always). The 10-item Trait Anger scale was used to assess the tendency to experience anger in a wide range of situations (e.g., "I have a fiery temper." "It makes me furious when I am criticized in front of others.”). The 8-item Anger-In subscale (e.g., "I am angrier than I am willing to admit.") was used to assess the tendency towards anger suppression. The STAXI has adequate convergent and discriminant validity and reliability (Spielberger, 1988). The two subscales also had adequate internal consistency in this sample (Cronbach’s alpha ranging from 0.79 to 0.84 ).

\subsection{Data Analysis}

Structural equation modeling with full information maximum-likelihood method using IBM SPSS AMOS ver.22 examined the relationships between mindfulness, anger rumination, anger-in, and trait anger in a large crosssectional sample.

Goodness of fit of the models to the data was evaluated with Chi-square statistic, comparative fit index (CFI), and root-mean-square error of approximation (RMSEA). A model is considered to have a good fit when the RMSEA is below 0.06 and the CFI is above 0.95 (Hu \& Bentler, 1999).

\section{Results}

Means, standard deviations, maximum, minimum, and alpha coefficients for the variables utilized in each analysis can be found in Table 1. The correlations between all four variables are displayed in Table 2. Although mindfulness was negatively correlated with anger rumination and trait anger, anger-in was not correlated with mindfulness. Anger rumination was positively correlated with both anger-in and trait anger.

To better understand the combined influence of mindfulness on anger rumination, anger-in, and trait anger, a model was run with the four variables (see Figure 1). The model included direct paths from mindfulness to anger rumination, anger-in, and trait anger and indirect paths from mindfulness though anger rumination to anger-in and trait anger. As expected, the model resulted in a fair fit to the data: $\chi^{2}=1.02, \mathrm{df}=1, p=0.31$; CFI $=1.00$, RMSEA $=0.01$ (95\% confidence interval, CI0.00, -0.14 ). This model included a significant direct effect from

Table 1. Descriptive statistics of study variables.

\begin{tabular}{cccccc}
\hline & Mean & SD & Minimum & Maximum & $\alpha$ \\
\hline Mindfulness & 113.19 & 12.06 & 80.00 & 155.00 & 0.78 \\
Anger Rumination & 37.86 & 11.97 & 19.00 & 76.00 & 0.94 \\
Anger-In & 21.46 & 4.15 & 8.00 & 32.00 & 0.79 \\
Trait Anger & 22.73 & 5.04 & 10.00 & 40.00 & 0.84 \\
\hline
\end{tabular}

Note: Analysis includes 366 participants.

Table 2. Zero-order correlations for study variables.

\begin{tabular}{ccccc}
\hline & 1 & 2 & 3 & 4 \\
\hline Mindfulness & - & & & \\
Anger Rumination & $-0.29^{* * *}$ & - & - & - \\
Anger-In & -0.08 & $0.27^{* *}$ & $0.12^{*}$ & - \\
Trait Anger & $-0.23^{* *}$ & $0.47^{* *}$ & $0.01,0<0.001$. \\
\hline
\end{tabular}

Note: Analysis includes 366 participants. ${ }^{*} p<0.05,{ }^{* *} p<0.001,{ }^{* * * *} p<0.001$. 


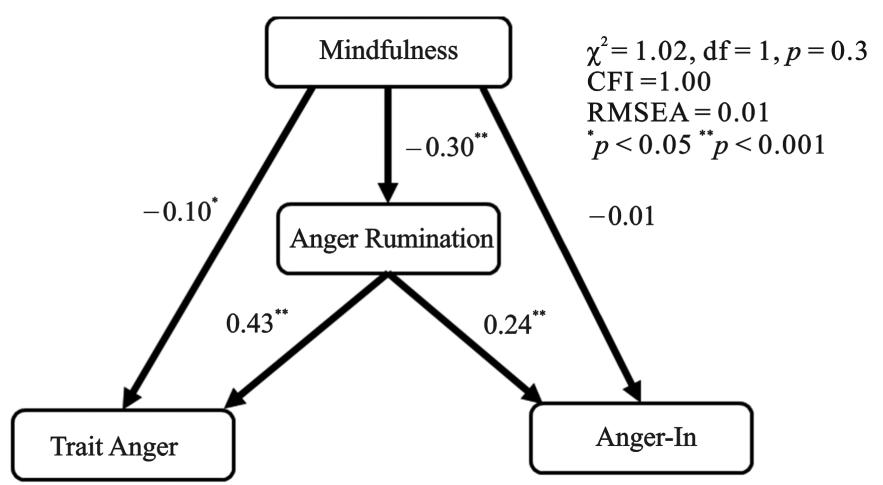

Figure 1. Mediating role of anger rumination in the associations between mindfulness, anger-in, and trait anger.

mindfulness to anger rumination $[\mathrm{b}=-0.30, \mathrm{SE}=0.04 ; 95 \% \mathrm{CI}-0.39,-0.22 ; p<0.001]$ and trait anger $[\mathrm{b}=$ $-0.10, \mathrm{SE}=0.05 ; 95 \% \mathrm{CI}-0.19,-0.01 ; p<0.05]$, but not to anger-in $[\mathrm{b}=-0.01, \mathrm{SE}=0.05 ; 95 \% \mathrm{Cl}-0.11,0.10$; $p=0.96]$. There were also significant direct effects from anger rumination to anger-in $[\mathrm{b}=0.24, \mathrm{SE}=0.05$; 95\%CI0.13, 0.34; $p<0.001]$ and trait anger $[\mathrm{b}=0.43, \mathrm{SE}=0.04 ; 95 \% \mathrm{CI}=0.34,0.50 ; p<0.001]$. Additionally, there were significant indirect effects from mindfulness through anger rumination to anger-in $[\mathrm{b}=-0.07, \mathrm{SE}=$ $0.02 ; 95 \% \mathrm{CI}-0.12,-0.04 ; p<0.001]$ and to trait anger $[\mathrm{b}=-0.13$, SE $=0.02 ; 95 \% \mathrm{CI}-0.17,-0.09 ; p<0.001]$.

\section{Discussion}

Three hypothesis that were (1) mindfulness will reduce anger rumination, (2) anger rumination will facilitate anger-in and trait anger, and (3) mindfulness will indirectly alleviate anger-in and trait anger through reducing anger rumination were verified.

Similar to Borders et al. (2010), mindfulness reduced rumination. The current study, however, showed that mindfulness also reduced anger rumination, which is more specific to anger problems. Borders et al. (2010) used the Rumination and Reflection Questionnaire (Trapnell \& Campbell, 1999) that assesses more general and non-emotional aspects of rumination by focusing on self-attentiveness (e.g., "I always seem to be rehashing in my mind recent things I've said or done.”), while anger rumination is considered a more specific aspect of rumination focusing on anger experience (e.g., "Whenever I experience anger, I keep thinking about it for a while.” Sukhodolsky et al., 2001). Given the difference in rumination compared to previous studies, the current findings indicate that mindfulness is not only effective for general rumination but also for anger rumination.

Additionally, anger rumination facilitated anger-in and trait anger. Although this study showed similar findings to Martin \& Dahlen (2005) and Owen (2011), the ARS to assess anger aspects of rumination has not been used in previous studies and suggests that anger rumination as well as general rumination facilitated anger-in and trait anger.

Mindfulness can be used in clinical practice with individuals who present with anger-related problems, such as anger-in and trait anger (Diebold, 2003). It was shown that mindfulness has indirect effects on anger-in and trait anger via reducing anger rumination. This study suggests not only that mindfulness may alleviate anger problems but also that anger rumination may have a mediating role in the associations between mindfulness, anger-in, and trait anger. Although the mediating role of anger rumination was discussed in Wright et al. (2009), it was not based on data. The current study verifies the assumptions by Wright et al. (2009) and suggests that anger rumination may be a key factor in treating problems of anger-in and trait anger.

\section{Conclusion}

The purpose of this study was to investigate whether mindfulness decreases anger-in and trait anger through anger rumination by utilizing structural equation modeling to examine the relationships between these variables.

The results of this study revealed that (1) mindfulness reduced anger rumination, (2) anger rumination facilitated anger-in and trait anger, and (3) mindfulness indirectly alleviated anger-in and trait anger through reducing anger rumination. 
There were some limitations in our study. First, the study was conducted using a cross-sectional design. An experimentation or intervention study would provide better understanding for the effects of mindfulness on anger-in and trait anger. Second, the generalizability of this study is limited by its focus on a student sample. We further need to compare differences between student and clinical samples.

Despite these limitations, this study provides initial evidence that mindfulness might be effective in modifying anger-in and trait anger, and that anger rumination plays an important role in this change. Although this model needs to be further tested in a clinical sample, the data from this study indicate that mindfulness can be a treatment option for anger-in and trait anger. If mindfulness is effective for reducing anger-in and trait anger through anger rumination, therapists should focus on reducing anger rumination with their clients to further enhance clinical outcomes.

\section{Acknowledgements}

This study was supported by the Foundation for Nagano Prefecture for Promoting Science.

\section{References}

Baer, R. A., Smith, G. T., Hopkins, J., Krietemeyer, J., \& Toney, L. (2006). Using Self-Report Assessment Methods to Explore Facets of Mindfulness. Assessment, 13, 27-45. http://dx.doi.org/10.1177/1073191105283504

Borders, A., Earleywine, M., \& Jajodia, A. (2010). Could Mindfulness Decrease Anger, Hostility, and Aggression by Decreasing Rumination? Aggressive Behavior, 36, 28-44. http://dx.doi.org/10.1002/ab.20327

Diebold, J. C. (2003). Mindfulness in the Machine: A Mindfulness-Based Cognitive Therapy for the Reduction of Driving Anger. Dissertation Abstracts International, 64, 5212.

Hatta, T., Ohbuchi, K., \& Hatta, J. (2013). Development of Japanese Version of Anger Rumination Scale. Japanese Journal of Applied Psychology, 38, 231-238.

Hu, L., \& Bentler, P. M. (1999). Cutoff Criteria for Fit Indexes in Covariance Structure Analysis: Conventional Criteria versus New Alternatives. Structural Equation Modeling: A Multidisciplinary Journal, 6, 1-55. http://dx.doi.org/10.1080/10705519909540118

Jorgensen, R. S., \& Kolodziej, M. E. (2007). Suppressed Anger, Evaluative Threat, and Cardiovascular Reactivity: A Tripartite Profile Approach. International Journal of Psychophysiology, 66, 102-108. http://dx.doi.org/10.1016/j.ijpsycho.2007.03.015

Kabat-Zinn, J. (1994). Wherever You Go, There You Are: Mindfulness Meditation in Everyday Life. New York: Hyperion Books.

Martin, R. C., \& Dahlen, E. R. (2005). Cognitive Emotion Regulation in the Prediction of Depression, Anxiety, Stress, and Anger. Personality and Individual Differences, 39, 1249-1260. http://dx.doi.org/10.1016/j.paid.2005.06.004

Nolen-Hoeksema, S., \& Morrow, J. (1991). A Prospective Study of Depression and Distress Following a Natural Disaster: The 1989 Lorma Prieta Earthquake. Journal of Personality and Social Psychology, 61, 105-121. http://dx.doi.org/10.1037/0022-3514.61.1.115

Lane, J. D., Seskevich, J. E., \&Pieper, C. F. (2007). Brief Meditation Training Can Improve Stress and Negative Mood. Alternatives Therapies in Health and Medicine, 13, 38-44.

Owen, J. M. (2011). Transdiagnostic Cognitive Processes in High Trait Anger. Clinical Psychology Review, 31, $193-202$. http://dx.doi.org/10.1016/j.cpr.2010.10.003

Silva, J. (2007). Mindfulness Based Cognitive Therapy for the Reduction of Anger in Married Men. Unpublished Doctorial Dissertation, New York: Hofstra University.

Spielberger, C. D. (1988). Manual for the State-Trait Anger Expression Inventory. Odessa, FL: Psychological Assessment Resources.

Spielberger, C. D. (1999). Manual for the State-Trait Anger Expression Inventory-2. Odessa, FL: Psychological Assessment Resources.

Sugiura, Y., Sato, A., Ito, Y., \& Murakami, H. (2012). Development and Validation of the Japanese Version of the Five Facet Mindfulness Questionnaire. Mindfulness, 3, 85-94. http://dx.doi.org/10.1007/s12671-011-0082-1

Sukhodolsky, D. G., Golub, A., \& Cromwell, E. N. (2001). Development and Validation of the Anger Rumination Scale. Personality and Individual Differences, 31, 689-700. http://dx.doi.org/10.1016/S0191-8869(00)00171-9

Suzuki, T., \& Haruki, Y. (1994). The Relationship between Anger and Circulatory Disease. Japanese Journal of Health Psychology, 7, 1-13. 
Trapnell, P. D., \& Campbell, J. D. (1999). Private Self-Consciousness and the Five-Factor Model of Personality: Distinguishing Rumination from Reflection. Journal of Personality Social Psychology, 76, 284-304.

http://dx.doi.org/10.1037/0022-3514.76.2.284

Trew, J. L., \& Alden, L. E. (2009). Predicting Anger in Social Anxiety: The Mediating Role of Rumination. Behaviour Research and Therapy, 47, 1079-1084. http://dx.doi.org/10.1016/j.brat.2009.07.019

Wright, S., Day, A., \& Howells, K. (2009). Mindfulness and the Treatment of Anger Problems. Aggression and Violent Behavior, 14, 396-401. http://dx.doi.org/10.1016/j.avb.2009.06.008 